\title{
Self-Monitoring and Psychoeducation in Bipolar Patients with a Smart-phone application (SIMPLe) project: Preliminary results from a feasibility study
}

\author{
Diego Hidalgo-Mazzei \\ Bipolar disorder unit, Department of \\ Psychiatry and Psychology, Institute \\ of Neuroscience, Hospital Clinic, \\ University of Barcelona, IDIBAPS, \\ CIBERSAM, Barcelona, Catalonia, \\ Spain
}

Tel. (34) 932275400 dahidalg@clinic.ub.es

\author{
Eduard Vieta \\ Bipolar disorder unit, Department of \\ Psychiatry and Psychology, Institute \\ of Neuroscience, Hospital Clinic, \\ University of Barcelona, IDIBAPS, \\ CIBERSAM, Barcelona, Catalonia, \\ Spain
}

Tel. (34) 932275400

evieta@clinic.ub.es

\begin{abstract}
Francesc Colom
Bipolar disorder unit, Department of Psychiatry and Psychology, Institute of Neuroscience, Hospital Clinic, University of Barcelona, IDIBAPS, CIBERSAM, Barcelona, Catalonia, Spain
\end{abstract}

Tel. (34) 932275400

fcolom@clinic.ub.es

\begin{abstract}
INTRODUCTION: The SIMPLe project was designed with the objective of developing a smartphone application, which could monitor and psychoeducate bipolar disorder patients through highly personalized messages based on both passive and active information. A secondary long-term objective of the SIMPLe project is to integrate biomedical information with the mobile behavior information collected in order to determine predictive relapses patterns. METHODS: The phases of the SIMPLe project involved an iterative process with the active participation of both patients and mental health professionals. The feasibility study phase of the project analyzed variables from the recruitment phase and server requests from the users' application. RESULTS: The participation in the feasibility study was offered to 70 stable bipolar patients.43 subjects were enrolled in the study. The main reasons for those who did not take part of the study was not having a compatible device(59\%). 95\% of the initial participants remain actively using the application during the 3 months considered during which no relapses were identified. CONCLUSIONS: The results so far suggest a high acceptance from the patients enrolled in the study. However, due to the fragmentation of the smartphone market, there is a risk that this sample would not represents the whole population of bipolar patients. DISCUSSION: Mobile technologies seems a feasible option, which could bring a novel type of data to the mental health field. Even though, there are still challenges ahead in order to harness its full potential.
\end{abstract}

\section{Categories and Subject Descriptors}

J.3 [Life and Medical sciences]: Health

\section{General Terms}

Reliability

\section{Keywords}

Mobile health; Feasibility; Bipolar disorder; Psychoeducation; Monitoring; Smartphones; Self-management.

\section{INTRODUCTION}

Bipolar disorder is a frequent condition in the general population with a high morbimortality [5]. It is characterized by dysfunctional episodic fluctuations between different mood phases ranging from depression to manic episodes with patients presenting frequent interepisodic subsyndromal symptoms. Frequently, people with this condition have a lack of insight about their diagnosis and symptoms, especially regarding manic phases, which leads to poor prognosis [2]. Besides the pharmacological treatment, adjunctive psychological interventions have shown to improve the long-term outcome of the disorder [16], although, taking into account the limited resources currently available, their extended implementation is still difficult and costly [14].

Among psychotherapeutic interventions, psychoeducational programs have proven to be cost-effective in helping patients recognize early signs or symptoms and adopt behavioral measures to prevent full-blown episodes, which are frequently associated with a high morbidity and more hospital admissions. Accordingly, there is an increased need to make this intervention more widely available, without compromising its quality [16].

On the other hand, mobile devices (i.e. smartphones, wearables) with hundreds of sensors and powerful processors are carried ubiquitously all day long by more and more subjects, with multiple and seamless purposes. The huge amount of information provided by these devices is of paramount importance to collect an essential part of the current Big Data, gradually leaving behind personal computers usage. Most importantly, from the perspective of mental health, the interaction between users and mobile devices can give deep insights about the subjects' behavior, which could be transmitted, almost instantly, through the Internet to dynamic cloud servers [22]. It seems that mental health patients are similarly adopting these new technologies in the same fashion as the general population [23].

These new mobile technologies are providing an Ecological Momentary Assessment (EMA), real-time and continuous data recollection on the subject's own environment [24]. Additionally, 
users' interactions with their mobile devices and their respective sensors could provide passive, objective information about their behavior [8]. Therefore, significant behavioral patterns can be inferred from all this kind of data in real-time, something which has the potential to design new prevention strategies as well as allowing personalized treatments [9]. Moreover, integrating behavioral and biomedical data (i.e. genetics, biomarkers, neuroimaging, etc.) and analyzing these datasets through adequate statistical methods and validation process, could ultimately provide new predictive models, previously unsuspected etiopathogenic factors and, at last, new treatment targets [13].

In addition, there is an increasing general consumers' preference to apply mobile technologies and the data they can offer into their own personal healthcare[6]. Physical activity, sleep patterns and location tracking data sets, are easily obtained from either increasingly cheaper smartphones or discrete new wearables [15]. Big technological corporations did not waste this opportunity, offering a significant number of devices and cloud services, which could store and integrate all the health data generated (e.g. Apple's Health, Google Fit, etc). Likewise, these some of the companies such as Apple and Google grew progressively interested in health research through their promising platforms Apple research kit and Google Study kit, respectively [4].

Considering the aforementioned background, the aim of the SIMPLe (Self-Monitoring and Psychoeducation in Bipolar Patients with a Smart-phone application) project was to develop and clinically validate a smartphone application to monitor symptoms and signs in bipolar patients, offering customized embedded psychoeducation contents and empower the self-management of their disorder in order to prevent relapses and hospitalizations. The project was based on a face-to-face group program, which has an increasing scientific evidence of its efficacy and cost-effectiveness reducing bipolar disorder relapses [7, 17]. The phases of the SIMPLe project involved an iterative process with the active participation of both patients and mental health professionals in the following order: 1. App development, 2. Feasibility study, and finally, after further improvements, 3. a Randomized Clinical Trial (RCT) assessing relapses prevention. A secondary long-term objective of the SIMPLe project is to integrate clinical correlates, biomarkers and genetics with the mobile behavior information collected in order to determine predictive relapses patterns[11]. We present in this paper preliminary results of the feasibility study.

\section{METHODS}

The feasibility study was conducted from March 2015 to June 2015. The participation in the study was proposed to a consecutive sample of adult patients diagnosed of bipolar disorder I, II or NOS (Not otherwise specified) attending the outpatient mental health clinic of the Hospital Clinic of Barcelona, Spain. Their usual psychiatrist explained briefly the aim of the study and offered an invitation. If the patient agreed to participate an informed consent was handed out and signed, if not, the psychiatrist recollected the age, sex, socioeconomic status and reasons given for not participating. For details about the methodology employed for the others phases of the project please refer to the published protocols [11].

The smartphone application employed (i.e. SIMPLe 1.0) for the study was available for smartphones with Android Operating System 4.0 or higher (Fig.1). The application requested the user through notifications to answer daily short screening tests about their mood symptoms and a weekly more comprehensive YES or NO test. Among the questions of the weekly test there was one about suicidal thoughts. In the case the patient answered affirmatively, an automatic alert email was sent to the mental healthcare team and the patient was offered to call the emergency services from their smartphones. If relevant clinical variations were detected in the score of the daily test by an algorithm, the user was requested to take an extra week test. A home screen reflected the progression of the mood symptoms alongside warning signs. Based on the answers given by the users, a daily psychoeducational message was received according to their specific state. Further specifications of the SIMPLe application 1.0 are described at the studies protocol [10].



Fig.1 The figure shows three screen-captures of the SIMPLe 1.0 application running on an Android Smartphone.

\section{RESULTS}

The participation in the study was offered to 70 stable bipolar patients. Forty-three $(61.4 \%)$ subjects with a mean age of 42.5 years accepted and were enrolled in the study. Those who did not take part of the study had a similar mean age of 45.7 years and the main reasons for not participating were: Not having a compatible cell phone (59\%), Not willing to monitor their mental health through an application (33\%), privacy and confidentiality concerns (4\%) and not having the knowledge to operate a smartphone (4\%). Among the subjects with incompatibility issues (16), 7 had IPhones, 3 had non-compatible Android phones, 2 had a Windows Phone and 4 did not had a smartphone at all. All the patients included in the study reported having an average medium or low income.

Included patients reported using their smartphones for a myriad of purposes, but the most common use was texting (Fig. 2).

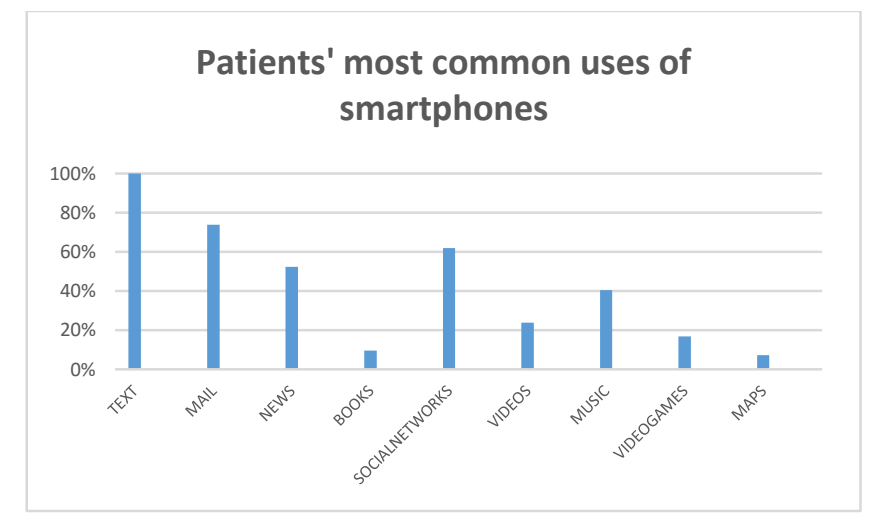

Fig.2 The figure shows the percentage of smartphone uses reported by the participants of the study. 
Two patients did not finally activate the application after the first interview, or used it only once and were considered early drop-outs. Among the active participants, 65.9\% ( $\mathrm{N}=27)$ were diagnosed with type I bipolar disorder, $24.4 \%(\mathrm{~N}=10)$ type II bipolar disorder and $9.8 \%(\mathrm{~N}=4)$ NOS. $56.8 \%$ were male and $43.2 \%$ were female. The significant percentage of the patients had a high educational level $40.5 \%$ while $40.5 \%$ and $16.2 \%$ had medium and low levels, respectively. Since the day the patients were enrolled in the study, the rate of completed daily tests were 0.74 per day and 1.13 per week. A total of 9 emergency alerts were received through the application and notified to the reference patients' psychiatrists. 95\% of the initial participants remain actively using the application during the 3 months considered during which no relapses were identified. Satisfaction interviews are due to take place on September 2015.

\section{DISCUSSION}

Our preliminary results suggest the initial main objectives of our project (i.e. develop and test the SIMPLe app with patients in a real clinical setting to evaluate its feasibility) were partially reached based on the high percentage of retention. However, data on the satisfaction of users are still pending. Similar smartphones applications for bipolar patients but with other aims reached comparable rates of users retention [8]. One of the main reasons we speculate could have helped to accomplish these objectives was the approach initially adopted which involved the patients throughout the development process. This approach have been reported to increase the percentage and satisfaction of bipolar patients using online psychological interventions [20,21]. Additional studies will evaluate the real clinical outcome impact using the smartphone application (i.e. SIMPLe 2.0) as well as the cost-efficiency of this approach in healthcare.

On the other hand, the second objective of our project seems that would require further workarounds in order to have satisfactory results. The reason being that the market fragmentation of current mobile Operating Systems (OSs) (Google's Android, Apple's iOS, Microsoft's Windows Phone, RIM's Blackberry, etc.) and smartphones ownership represents a still unaddressed and growing issue in the field which could have a direct impact on any project employing mobile technologies.

Even when the sample is small, our results support the global results and surveys from larger samples and further increase awareness of the fragmentation and stratification issues in health projects relying in mobile.

According to ITU in 2015, around $95 \%$ of the worldwide population have a subscription for a mobile-cellular telephone, but despite this rapid growth, less than $50 \%$ of these telephones are connected to the Internet [12]. Moreover, among smartphones users, Android and iOS together have a $96.4 \%$ of smartphones market share according to IDC, $78 \%$ of this amount pertaining to Android [18].

Hence, the promising future of the components of Big Data provided by mobile devices research might be severely hampered by the companies' aim of selling their own products with data collected through non-open platforms. In other words, any smartphone or wearable provides relevant information to databases owned by these companies, not available for being exported or integrated with clinical information [3]. The upcoming wave of Internet of Things (IoT) and its potential valuable application in health, may further broaden the gap among these datasets if there are no firm initiatives to systematically integrate them [19].

Nevertheless, in the hypothetical instance that technical, sociological and ethical issues could be tackled, and most importantly, researchers could have fully available access to these diverse and vast open source big Datasets, the remaining challenge will be how to adequately analyze and process this kind of data to reach valid and meaningful scientific conclusions[1].

\section{CONCLUSIONS}

The results so far suggest a high acceptance and adoption from the patients enrolled in the study using our mobile mental health application based on the rates of usage and retention. However, due to the fragmentation of the smartphone market, there is a risk that this sample would not represent the whole population of bipolar patients in order to have a representative Big Data for research aims. Overall, all these issues could be overcome on the long run, only if there is a true intent of public and private sectors to altruistically collaborate towards an open health science, which could ultimately improve health research and as a result have a prompt impact in public health as well.

\section{ACKNOWLEDGMENTS}

Indirectly supported by an Emili Letang grant from the Hospital Clínic of Barcelona (to DH). Dr Colom is funded by the Spanish Ministry of Economy and Competitiveness, Instituto Carlos III, through a "Miguel Servet II" postdoctoral contract and a FIS (PI 12/00910). The SIMPLe project is supported by research grants from the Ministry of Economy and Competitiveness, Instituto Carlos III (PI14/00286 and PI15/00588). SIMPLe app. All copyrights are reserved (C) 2015.

\section{REFERENCES}

[1] Alyass, A. et al. 2015. From big data analysis to personalized medicine for all: challenges and opportunities. BMC Medical Genomics. 8, 1 (Jun. 2015), 33.

[2] Bonnín, C. del M. et al. 2014. Verbal memory as a mediator in the relationship between subthreshold depressive symptoms and functional outcome in bipolar disorder. Journal of affective disorders. 160, (May 2014), $50-4$.

[3] Boulos, M.N.K. et al. 2011. How smartphones are changing the face of mobile and participatory healthcare: an overview, with example from eCAALYX. Biomedical engineering online. 10, 1 (Jan. 2011), 24.

[4] Campbell, K.R. 2015. An apple a day: changing medicine through technology and engagement. Future Cardiology. 11, 3 (2015), 259-260.

[5] Catalá-López, F. et al. 2013. The increasing burden of mental and neurological disorders. European neuropsychopharmacology: the journal of the European 
College of Neuropsychopharmacology. 23, 11 (Nov. 2013), 1337-9.

[6] Chiauzzi, E. et al. 2015. Patient-centered activity monitoring in the self-management of chronic health conditions. Jama. 313, 5 (2015), 77.

[7] Colom, F. et al. 2009. Group psychoeducation for stabilised bipolar disorders: 5-year outcome of a randomised clinical trial. The British journal of psychiatry: the journal of mental science. 194, 3 (Mar. 2009), 260-5.

[8] Faurholt-Jepsen, M. et al. 2014. Daily electronic monitoring of subjective and objective measures of illness activity in bipolar disorder using smartphones; the MONARCA II trial protocol: a randomized controlled single-blind parallel-group trial. BMC psychiatry. 14, 1 (Nov. 2014), 309.

[9] Glenn, T. and Monteith, S. 2014. New measures of mental state and behavior based on data collected from sensors, smartphones, and the Internet. Current psychiatry reports. 16, 12 (Dec. 2014), 523.

[10] Hidalgo-Mazzei, D. et al. 2014. Self-Monitoring and Psychoeducation in Bipolar Patients With a Smart-phone Application (SIMPLe). ClinicalTrials.gov Identifier: NCT02258711. 2014.

[11] Hidalgo-Mazzei, D. et al. 2015. Self-monitoring and psychoeducation in bipolar patients with a smart-phone application (SIMPLe) project: design, development and studies protocols. BMC Psychiatry. 15, 1 (Mar. 2015), 52.

[12] International Telecommunication Union 2015. ITU ICT Facts and Figures - The world in 2015.

[13] McIntyre, R.S. et al. 2014. Advancing biomarker research: utilizing "Big Data" approaches for the characterization and prevention of bipolar disorder. Bipolar disorders. 16, 5 (Aug. 2014), 531-47.

[14] Miklowitz, D.J. and Scott, J. 2009. Psychosocial treatments for bipolar disorder: cost-effectiveness, mediating mechanisms, and future directions. Bipolar disorders. 11 Suppl 2, (Jun. 2009), 110-22.

[15] Patel, M.S. et al. 2015. Wearable Devices as Facilitators, Not Drivers, of Health Behavior Change. JAMA. 313, 5 (Jan. 2015), 459-460.

[16] Reinares, M. et al. 2014. Psychosocial interventions in bipolar disorder: what, for whom, and when. Journal of affective disorders. 156, (Mar. 2014), 46-55.
[17] Scott, J. et al. 2009. Long-term mental health resource utilization and cost of care following group psychoeducation or unstructured group support for bipolar disorders: a cost-benefit analysis. The Journal of clinical psychiatry. 70, 3 (Mar. 2009), 378-86.

[18] Smartphone OS Market Share, Q1 2015: 2015. http://www.idc.com/prodserv/smartphone-os-marketshare.jsp. Accessed: 2015-04-07.

[19] Suciu, G. et al. 2015. Big Data, Internet of Things and Cloud Convergence for E-Health Applications. New Contributions in Information Systems and Technologies. A. Rocha et al., eds. Springer International Publishing. 151-160.

[20] Todd, N.J. et al. 2014. A web-based self-management intervention for Bipolar Disorder "Living with Bipolar": A feasibility randomised controlled trial. Journal of affective disorders. 169, (Dec. 2014), 21-9.

[21] Todd, N.J. et al. 2013. What do service users with bipolar disorder want from a web-based self-management intervention? A qualitative focus group study. Clin Psychol Psychother. 20, 6 (2013), 531-543.

[22] Torous, J. et al. 2015. Realizing the Potential of Mobile Mental Health: New Methods for New Data in Psychiatry. Current psychiatry reports. 17, 8 (Aug. 2015), 602.

[23] Torous, J. et al. 2014. Smartphone ownership and interest in mobile applications to monitor symptoms of mental health conditions. JMIR mHealth and uHealth. 2, 1 (Jan. 2014), e2.

[24] Wenze, S.J. et al. 2014. Feasibility and Acceptability of a Mobile Intervention to Improve Treatment Adherence in Bipolar Disorder: A Pilot Study. Behavior modification. 38, 4 (Jan. 2014), 497-515. 